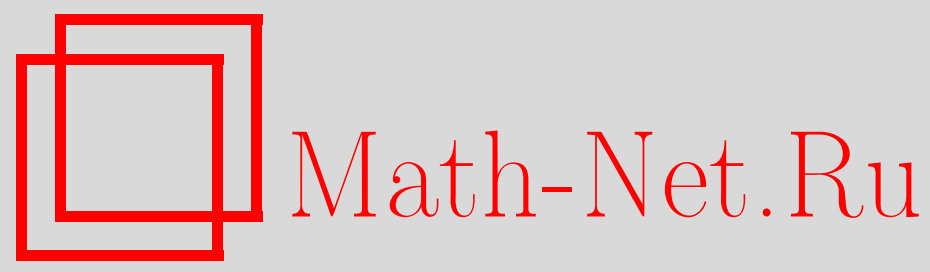

О. В. Бесов, О продолжении нулем функций многих переменных, Матем. заметки, 1998, том 64, выпуск 3, 351-365

DOI: https://doi.org/10.4213/mzm1405

Использование Общероссийского математического портала Math-Net.Ru подразумевает, что вы прочитали и согласны с пользовательским соглашением http://www.mathnet.ru/rus/agreement

Параметры загрузки:

IP: 54.89 .56 .158

26 апреля 2023 г., 05:14:09

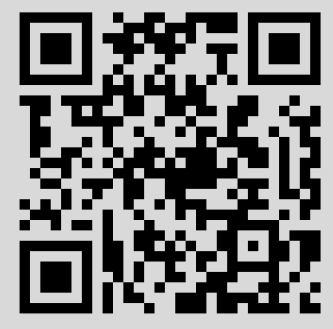




\title{
О ПРОДОЛЖЕНИИ НУЛЕМ ФУНКЦИЙ МНОГИХ ПЕРЕМЕННЫХ
}

\author{
О.В. Бесов
}

\footnotetext{
Устанавливаются достаточные условия на область $G \subset \mathbb{R}^{n}$ для возможности продолжения нулем на $\mathbb{R}^{n}$ заданных на $G$ функций с сохранением гладкости в интегральной норме.

Библиография: 15 названий.
}

Пусть $1 \leqslant p<\infty, 0<s<1 / p$ и при некотором $M>0$ числовая функция $f \in L_{p}((0, \infty))$ обладает свойствами

$$
\left\|f \mid L_{p}((0, \infty))\right\| \leqslant M, \quad\left(\int_{0}^{\infty}|f(x+h)-f(x)|^{p} d x\right)^{1 / p} \leqslant M h^{s}, \quad h \in(0,1] .
$$

Тогда, как показано Кутнером [1],

$$
\left(\int_{0}^{h}|f(x)|^{p} d x\right)^{1 / p} \leqslant C M h^{s}, \quad h \in(0,1]
$$

при некотором $C>0$, не зависящем от $f, M$ и $h$. Отсюда следует [1], очевидно, что для этой функции $f$ после ее продолжения нулем на $(-\infty, 0]$ (с сохранением обозначения $f$ ) будет вьполняться оценка

$$
\left(\int_{-\infty}^{\infty}|f(x+h)-f(x)|^{p} d x\right)^{1 / p} \leqslant C_{1} M h^{s}, \quad h \in(0,1]
$$

где $C_{1}$ не зависит от $f, M, h$. Этот результат (о возможности продолжения нулем с сохранением гладкости) был обобщен С. М. Никольским [2] на случай функций, заданных в ограниченной области $G \subset \mathbb{R}^{n}$ с непрерьвно дифференцируемой $(n-1)$-мерной гранищей $\partial G$.

Возможность продолжения нулем с полуоси на ось для пространств $B_{p p}^{s}((0, \infty))$, $0<s<1 / p$, установлена Г. Н. Яковлевым [3] и в эквивалентной форме В.А. Солонниковым [4]. Г. А. Калябин [5] установил возможность продолжения нулем функций пространств $B_{p q}^{s}(G), F_{p q}^{s}(G), 0<s<1 / p$, для области $G \subset \mathbb{R}^{n}$ с липшицевой $(n-1)$-мерной гранищей.

Работа выполнена при финансовой поддержке Российского фонда фундаментальных исследований, грант № 96-01-00243, и программы “Ведущие научные школы”, грант № 96-15-96102. 
Мы рассматриваем здесь области $G \subset \mathbb{R}^{n}$ со свойством гибкого конуса и устанавливаем достаточные условия для возможности продолжения нулем функций из пространств $B_{p q}^{s}(G), F_{p q}^{s}(G)$ соответственно до функций из $B_{p q}^{s}\left(\mathbb{R}^{n}\right), F_{p q}^{s}\left(\mathbb{R}^{n}\right)$.

В работе [5] Калябин строит сначала оператор продолжения (методом Стейна), а затем решает вопрос продолжения нулем, сводя его к ограниченности оператора умножения на характеристическую функцию области $G$. В нашей ситуации существование оператора продолжения априори неизвестно, оно фактически устанавливается при доказательстве возможности продолжения нулем.

Введем следующие обозначения. Пусть $\mathbb{Z}-$ множество целых чисел; $\mathbb{N}$ - множество натуральных чисел; $\mathbb{N}_{0}=\mathbb{N} \cup\{0\} ; \mathbb{R}^{n}, n \in \mathbb{N}, n \geqslant 2,-n$-мерное евклидово пространство со стандартным базисом $\left\{e^{1}, \ldots, e^{n}\right\} ; x=\left(x_{1}, \ldots, x_{n}\right)=\sum_{i=1}^{n} x_{i} e^{i}=\left(x_{i}\right)$. Для $x, y \in \mathbb{R}^{n}$ пусть $x y=\left(x_{i} y_{i}\right),[x, y]$ - отрезок с концами в точках $x, y ; x \pm a E=\{y: y=$ $x \pm a z, z \in E\}\left(E \subset \mathbb{R}^{n}, a \in \mathbb{R}^{n}\right.$ или $\left.a \in \mathbb{R}^{1}\right) ; Q_{0}=[-1,1]^{n} ; G$ - область в $\mathbb{R}^{n}$, при $t>0 G(t)=\left\{x: x+t Q_{0} \subset G\right\} ; U_{t}(E)-t$-окрестность множества $E$, при $a \in \mathbb{R}^{n}$ $B_{t}(a)=U_{t}(a), B_{t}=B_{t}(0) ; \chi$ - характеристическая функция шара $B_{1}(0)$ или отрезка $[-1,1], \chi_{E}-$ характеристическая функция множества $E \subset \mathbb{R}^{n}$.

Для числовой функции $f, m \in \mathbb{N}_{0}, h \in \mathbb{R}^{1}$ положим

$$
\begin{aligned}
\Delta_{i}^{m}(h) f(x) & =\sum_{k=0}^{m}(-1)^{m-k}\left(\begin{array}{c}
m \\
k
\end{array}\right) f\left(x+k h e^{i}\right), \\
\Delta_{i}^{m}(h, G) f(x) & = \begin{cases}\Delta_{i}^{m}(h) f(x), & {\left[x, x+m h e^{i}\right] \subset G(|h|),} \\
0, & {\left[x, x+m h e^{i}\right] \not \subset G(|h|),}\end{cases} \\
\widetilde{\Delta}_{i}^{m}(h, G) f(x) & =\int_{-1}^{1}\left|\Delta_{i}^{m}(u h, G) f(x)\right| d u ;
\end{aligned}
$$

$C_{0}\left(\mathbb{R}^{n}\right)$ - пространство финитных непрерьвных на $\mathbb{R}^{n}$ функций; $L_{p}(G)$ - лебегово пространство функций $f: G \rightarrow \mathbb{R}^{1}$ с нормой

$$
\left\|f \mid L_{p}(G)\right\|=\|f\|_{p, G}=\left(\int_{G}|f(x)|^{p} d x\right)^{1 / p}, \quad 1 \leqslant p \leqslant \infty
$$

(при $p=\infty$ правая часть понимается как $\left.\operatorname{ess}_{\sup }|f|\right),\|f\|_{p}=\left\|f\left|L_{p}\|=\| f\right| L_{p}\left(\mathbb{R}^{n}\right)\right\|$; $L_{q}^{*}$ - пространство функций $f:\left(0, h_{0}\right) \rightarrow \mathbb{R}^{1}$ с нормой

$$
\left\|f \mid L_{q}^{*}\right\|=\left(\int_{0}^{h_{0}}|f(t)|^{q} \frac{d t}{t}\right)^{1 / q}, \quad h_{0}>0, \quad 1 \leqslant q<\infty .
$$

ОПРЕДЕЛЕНИЕ 1 (см. [6]). Область $G \subset \mathbb{R}^{n}$ назьвается областью со свойством гибкого конуса, если при некоторых $\delta_{0}>0, T>0$ для любого $x \in G$ существует путь

$$
\rho(t)=\left(\rho_{1}(t), \ldots, \rho_{n}(t)\right)=\rho(t, x), \quad 0 \leqslant t \leqslant T,
$$

со свойствами:

а) для всех $i \in\{1, \ldots, n\} \quad \rho_{i}(u)$ абсолютно непрерьвны на $[0, T],\left|\rho_{i}(u)\right| \leqslant 1$ для почти всех $u \in[0, T]$;

б) $\rho(0)=0, x+\bigcup_{0 \leqslant t \leqslant T}\left(\rho(t, x)+t \delta_{0} Q_{0}\right) \subset G$. 
ОПРЕДЕЛЕНИЕ 2 . Пусть область $G \subset \mathbb{R}^{n}, m>s>0, m \in \mathbb{N}, 0<h_{0} \leqslant 1$.

Пространствами $B_{p q}^{s}(G)(1 \leqslant p, q \leqslant \infty), F_{p q}^{s}(G)(1<p, q<\infty)$ назьваются банаховы пространства локально суммируемых на $G$ функций с нормами соответственно

$$
\left\|f\left|B_{p q}^{s}(G)\|=\| f\right| L_{p}(G)\right\|+\sum_{i=1}^{n}\left(\int_{0}^{h_{0}}\left\|\Delta_{i}^{m}(h, G) f\right\|_{p}^{q} h^{-s q} \frac{d h}{h}\right)^{1 / q}
$$

(при $q=\infty$ вместо $L_{q}$-нормы по мере $d h / h$ берется $L_{\infty}$-норма),

$$
\left\|f\left|F_{p q}^{s}(G)\|=\| f\right| L_{p}(G)\right\|+\sum_{i=1}^{n}\left\|\left(\int_{0}^{h_{0}}\left(\widetilde{\Delta}_{i}^{m}(h, G) f\right)^{q} h^{-s q} \frac{d h}{h}\right)^{1 / q}\right\|_{p} .
$$

Различные эквивалентные нормировки указанных пространств см., например, в [6]. В частности, нормы $B_{p q}^{s}(G), F_{p q}^{s}(G)$ остаются эквивалентньми при изменении $h_{0}$, а для области $G$ со свойством гибкого конуса и при изменении $m$.

ОПРЕДЕЛЕниЕ 3 . Пусть область $G \subset \mathbb{R}^{n}, s>0$. Через $\stackrel{\circ}{B}_{p q}^{s}\left(\mathbb{R}^{n}, G\right), 1 \leqslant p, q \leqslant \infty$ $\left(\stackrel{\circ}{F}_{p q}^{s}\left(\mathbb{R}^{n}, G\right), 1<p, q<\infty\right)$, будем обозначать замыкание в норме $B_{p q}^{s}\left(\mathbb{R}^{n}\right)\left(F_{p q}^{s}\left(\mathbb{R}^{n}\right)\right.$ соответственно) множества функций $f$, для которых $\operatorname{dist}\left(\operatorname{supp} f, \mathbb{R}^{n} \backslash G\right)>0$.

ОПРЕДЕЛЕНИЕ 4 . Пусть область $G \subset \mathbb{R}^{n}, s>0,0<h_{0} \leqslant 1$. Через $B_{p q}^{s}(G)$ $(1 \leqslant p, q \leqslant \infty)$ будем обозначать банахово пространство функций $f \in B_{p q}^{s}(G)$ с конечной нормой

$$
\left\|f\left|\stackrel{*}{B}_{p q}^{s}(G)\|=\| f\right| B_{p q}^{s}(G)\right\|+\left(\int_{0}^{h_{0}}\left\|f \mid L_{p}\left(G \cap U_{h}(\partial G)\right)\right\|^{q} h^{-s q} \frac{d h}{h}\right)^{1 / q}
$$

(при $q=\infty$ вместо $L_{q}$-нормы по мере $d h / h$ берется $L_{\infty}$-норма).

Через $\stackrel{*}{F}_{p q}^{s}\left(\mathbb{R}^{n}, G\right)(1<p, q<\infty)$ будем обозначать банахово пространство функций $f \in F_{p q}^{s}(G)$ с конечной нормой

$$
\left\|f\left|\stackrel{*}{F}_{p q}^{s}(G)\|=\| f\right| F_{p q}^{s}(G)\right\|+\left(\int_{G} \chi\left(\frac{r(x)}{h_{0}}\right)\left|\frac{f(x)}{r(x)^{s}}\right|^{p} d x\right)^{1 / p} .
$$

Легко видеть, что нормы $\ddot{B}_{p q}^{s}, \stackrel{*}{\mathscr{F}_{p q}}$ остаются эквивалентными при изменении $h_{0}$.

Символом $\mathscr{E}_{0}$ обозначим оператор, ставящий в соответствие определенной на $G$ функции ее продолжение нулем на $\mathbb{R}^{n}$.

Лемма 1. Пусть область $G \subset \mathbb{R}^{n}, s>0,1 \leqslant p \leqslant \infty, 1 \leqslant q<\infty$. Тогда onepamopbl

$$
\mathscr{E}_{0}: \stackrel{*}{B}_{p q}^{s}(G) \rightarrow \stackrel{\stackrel{B}{B}}{s q}_{p q}\left(\mathbb{R}^{n}, G\right), \quad \mathscr{E}_{0}: \stackrel{*}{B}_{p \infty}^{s}(G) \rightarrow B_{p \infty}^{s}\left(\mathbb{R}^{n}\right)
$$

ограничены. 
ДокАЗАТЕльСтво. Для $f \in \stackrel{*}{B}_{p q}^{s}(G)$ положим $f=\mathscr{E}_{0} f$ на $\mathbb{R}^{n}$. Тогда при некотором $C>0$

$$
\left\|f\left|B_{p q}^{s}\left(\mathbb{R}^{n}\right)\|\leqslant C\| f\right| \stackrel{*}{B_{p q}^{s}}(G)\right\|, \quad f \in B_{p q}^{s}(G),
$$

что следует из оценок

$$
\begin{aligned}
& \sum_{j=1}^{n}\left(\int_{0}^{h_{0}}\left\|\Delta_{j}^{m}\left(h, U_{(m+1) h}(\partial G)\right) f\right\|_{p}^{q} h^{-s q} \frac{d h}{h}\right)^{1 / q} \\
& \quad \leqslant C_{1}\left(\int_{0}^{h_{0}}\left\|f \mid L_{p}\left(G \cap U_{(m+1) h}(\partial G)\right)\right\|^{q} h^{-s q} \frac{d h}{h}\right)^{1 / q} \leqslant C_{2}\left\|f \mid \stackrel{*}{B}_{p q}^{s}(G)\right\| .
\end{aligned}
$$

Этим установлена ограниченность оператора $\mathscr{E}_{0}: \stackrel{*}{B}{ }_{p q}^{s}(G) \rightarrow B_{p q}^{s}\left(\mathbb{R}^{n}\right)$, причем доказательство сохраняется и при $q=\infty$.

Обозначим через $\widetilde{r}(x)$ регуляризованное расстояние от точки $x \in G$ до $\mathbb{R}^{n} \backslash G($ см. [7, п. 2.1]), доопределенное нулем на $\mathbb{R}^{n} \backslash G$. Отметим, что $\widetilde{r}(x) \in C^{\infty}(G), r(x) \leqslant \widetilde{r}(x) \leqslant 2 r(x)$ для $x \in G,\left|D^{\alpha} \widetilde{r}(x)\right| \leqslant C_{\alpha} \widetilde{r}(x)^{1-|\alpha|}$, для всех $\alpha$ и $x \in G$. Возможность построения $\widetilde{r}(x)$ с постоянной 2 (и даже с любой постоянной $1+\varepsilon>1$ ) в правой части двусторонней оценки установлена в [8].

Пусть $\eta \in C^{\infty}\left(\mathbb{R}^{1}\right), 0 \leqslant \eta \leqslant 1, \eta(t)=1$ при $t \leqslant 1 / 3, \eta(t)=0$ при $t \geqslant 2 / 3$. Рассмотрим при $\varepsilon>0$ функцию

$$
f_{(\varepsilon)}(x)=f(x) \eta_{\varepsilon}(\widetilde{r}(x))=f(x) \eta\left(\frac{\widetilde{r}(x)}{\varepsilon}\right) .
$$

Для доказательства леммы достаточно показать, что $\left\|f_{(\varepsilon)} \mid B_{p q}^{s}\left(\mathbb{R}^{n}\right)\right\| \rightarrow 0$ при $\varepsilon \rightarrow 0$. Для этого достаточно убедиться, что при $i=1, \ldots, n$

$$
\int_{0}^{h_{0}}\left\|\Delta_{i}^{2 m-1}(h) f_{(\varepsilon)}\right\|_{p}^{q} h^{-s q} \frac{d h}{h} \rightarrow 0 \quad \text { при } \varepsilon \rightarrow 0 .
$$

Заметим, что

$$
\left|\Delta_{i}^{2 m-1}(h) f_{(\varepsilon)}(x)\right| \leqslant C \sum_{k=0}^{2 m-1}\left|\Delta_{i}^{k}(h) f(x)\right| \cdot\left|\Delta_{i}^{2 m-1-k}(h) \eta_{\varepsilon}\left(\widetilde{r}\left(x+k h e^{i}\right)\right)\right| .
$$

Учитьвая ограниченность последней разности и ее оценку с помощью теоремы Лагранжа о среднем и полагая

$$
\bar{\eta}(t)=\sum_{k=1}^{2 m-1}\left|\eta^{(k)}(t)\right|, \quad \bar{\eta}_{\varepsilon}(t)=\bar{\eta}\left(\frac{t}{\varepsilon}\right)
$$

получаем

$$
\begin{aligned}
\left|\Delta_{i}^{2 m-1}(h) f_{(\varepsilon)}(x)\right| \leqslant & C_{1} \sum_{k=0}^{2 m-1}\left|\Delta_{i}^{k}(h) f(x)\right|\left(\frac{h}{\varepsilon}\right)^{2 m-1-k} \\
& \times \max _{v \in[k, 2 m-1]}\left(\left(\bar{\eta}_{\varepsilon}+\delta_{k, 2 m-1} \eta_{\varepsilon}\right)\left(\widetilde{r}\left(x+v h e^{i}\right)\right)\right),
\end{aligned}
$$


где $\delta_{k l}$ - символ Кронекера. В силу (3) при $0<h<\varepsilon$ имеем

$$
\begin{gathered}
\left\|\Delta_{i}^{2 m-1}(h) f_{(\varepsilon)}\right\|_{p} \leqslant C\left\|f\left|L_{p}\left(U_{2 m \varepsilon}(\partial G)\right)\left\|\left(\frac{h}{\varepsilon}\right)^{m}+C\right\| \Delta_{i}^{m}(h) f\right| L_{p}\left(U_{2 m \varepsilon}(\partial G)\right)\right\| \\
\int_{0}^{\varepsilon}\left\|\Delta_{i}^{2 m-1}(h) f_{(\varepsilon)}\right\|_{p}^{q} h^{-s q} \frac{d h}{h} \leqslant C_{1}\left\|f \mid L_{p}\left(U_{2 m \varepsilon}(\partial G)\right)\right\|^{q} \varepsilon^{-s q} \\
\quad+C \int_{0}^{\varepsilon}\left\|\Delta_{i}^{m}(h) f \mid L_{p}\left(U_{2 m \varepsilon}(\partial G)\right)\right\|^{q} h^{-s q} \frac{d h}{h} \rightarrow 0 \quad \text { при } \quad \varepsilon \rightarrow 0 .
\end{gathered}
$$

При $h>\varepsilon$

$$
\begin{gathered}
\left|\Delta_{i}^{2 m-1}(h) f_{(\varepsilon)}(x)\right| \leqslant 2^{2 m-1} \sum_{\nu=0}^{2 m-1}\left|f\left(x+\nu h e^{i}\right)\right|\left(\eta_{\varepsilon}+\bar{\eta}_{\varepsilon}\right)\left(\widetilde{r}\left(x+\nu h e^{i}\right)\right), \\
\int_{\varepsilon}^{h_{0}}\left\|\Delta_{i}^{2 m-1}(h) f_{(\varepsilon)}\right\|_{p}^{q} h^{-s q} \frac{d h}{h} \leqslant C \sum_{\nu=0}^{2 m-1} \int_{\varepsilon}^{h_{0}}\left\|f \mid L_{p}\left(G \cap U_{\varepsilon}\left(\partial G-\nu h e^{i}\right)\right)\right\|^{q} h^{-s q} \frac{d h}{h} \rightarrow 0
\end{gathered}
$$

при $\varepsilon \rightarrow 0$. Отсюда и из (4) следует (2) и утверждение леммы.

ЛЕмма 2. Пусть область $G \subset \mathbb{R}^{n}$ обладает свойством гибкого конуса, $s>0$, $1<p, q<\infty$. Тогда оператор

$$
\mathscr{E}_{0}: \stackrel{*}{F}_{p q}^{s}(G) \rightarrow \stackrel{\circ}{F}_{p q}^{s}\left(\mathbb{R}^{n}, G\right)
$$

ограничен.

ДокАЗАтЕльСтво. Рассмотрим в пространстве $F_{p q}^{s}(G)$ норму, эквивалентную [6], [9] норме из определения 2:

$$
\left\|f\left|F_{p q}^{s}(G)\left\|_{(1)}=\right\| f\right| L_{p}(G)\right\|+\left\|\left(\int_{0}^{h_{0}}\left(\widetilde{\Delta}^{m}(h, G) f(\cdot)\right)^{q} h^{-s q} \frac{d h}{h}\right)^{1 / q}\right\|_{p},
$$

где

$$
\begin{gathered}
\widetilde{\Delta}^{m}(h, G) f(x)=\int_{|y|<1}\left|\Delta^{m}(h y, G) f(x)\right| d y \\
\Delta^{m}(y, G) f(x)= \begin{cases}\Delta^{m}(y) f(x), & {[x, x+m y] \subset G(|y|),} \\
0, & {[x, x+m y] \not \subset G(|y|),}\end{cases} \\
\Delta^{m}(y) f(x)=\sum_{k=0}^{m}(-1)^{m-k}\left(\begin{array}{c}
m \\
k
\end{array}\right) f(x+k y) .
\end{gathered}
$$

Пусть $f \in \stackrel{*}{F}_{p q}^{s}(G)$. Положим $f=\mathscr{E}_{0} f$ на $\mathbb{R}^{n}$. Покажем, что

$$
\left\|f\left|F_{p q}^{s}\left(\mathbb{R}^{n}\right)\|\leqslant C\| f\right| \stackrel{*}{F}_{p q}^{s}(G)\right\|
$$

с постоянной $C$, не зависящей от $f$. Для этого достаточно установить, что

$$
\left\|N\left|L_{p}\left(U_{(m+1) h_{0}}(\partial G)\right)\|\leqslant C\| f\right| \stackrel{*}{F}_{p q}^{s}(G)\right\|
$$


где при $r(x)<(m+1) h_{0}$

$$
\begin{aligned}
N(x)^{q} & =\int_{r(x) /(m+1)}^{h_{0}}\left(\int_{r(x) /(h(m+1))<|y|<1}\left|\Delta^{m}(h y) f(x)\right| d y\right)^{q} h^{-s q} \frac{d h}{h} \\
& \leqslant C \sum_{\nu=0}^{m} \int_{r(x) /(m+1)}^{h_{0}}\left(\int_{r(x) /(m+1)<|z|<h}|f(x+\nu z)| d z\right)^{q} h^{-s q-n q} \frac{d h}{h} \\
& \leqslant C_{1}\left(\frac{|f(x)|^{q}}{r(x)^{s q}}+\int_{r(x) /(m+1)}^{h_{0}}\left(\int_{r(x) /(m+1)<|y|<m h}|f(x+y)| d y\right)^{q} h^{-s q-n q} \frac{d h}{h}\right) .
\end{aligned}
$$

С помощью неравенства Минковского имеем

$$
\begin{aligned}
N(x) & \leqslant C_{2} \frac{|f(x)|}{r(x)^{s}}+C_{2} \int_{r(x) /(m+1)<|y|<m h_{0}}|f(x+y)| \cdot|y|^{-s} \frac{d y}{|y|^{n}} \\
& \leqslant C_{2} \frac{|f(x)|}{r(x)^{s}}+C_{3} \int_{r(x) /(m+1)<|y|<m h_{0}} \frac{|f(x+y)|}{r(x+y)^{s}} \frac{d y}{|y|^{n} .}
\end{aligned}
$$

Теперь для доказательства (5) остается лишь воспользоваться ограниченностью оператора

$$
K_{0} g(x)=\int_{r(x)<|y|<T} g(x+y) \frac{d y}{|y|^{n}}
$$

действующего в $L_{p}\left(\mathbb{R}^{n}\right)$ (см. ниже следствие из лемм 3,4$)$.

Рассмотрим при $\varepsilon>0$ функцию $f_{(\varepsilon)}$ из $(1)$. Остается показать, что $\left\|f_{(\varepsilon)} \mid F_{p q}^{s}\left(\mathbb{R}^{n}\right)\right\| \rightarrow 0$ при $\varepsilon \rightarrow 0$, для чего достаточно установить, что для $i=1, \ldots, n$

$$
\left\|\left(\int_{0}^{h_{0}}\left(\widetilde{\Delta}_{i}^{2 m-1}(h) f_{(\varepsilon)}\right)^{q} h^{-s q} \frac{d h}{h}\right)^{1 / q} \mid L_{p}\left(\mathbb{R}^{n}\right)\right\| \rightarrow 0 \quad \text { при } \varepsilon \rightarrow 0 .
$$

Приведем необходимые оценки. Пусть сначала $0<h<\varepsilon$. Из (3) имеем

$$
\begin{aligned}
\widetilde{\Delta}_{i}^{2 m-1}(h) f_{(\varepsilon)}(x)= & \int_{-1}^{1}\left|\Delta_{i}^{2 m-1}(u h) f_{(\varepsilon)}(x)\right| d u \\
\leqslant & C\left(\sum_{k=0}^{0}+\sum_{k=1}^{m-1}+\sum_{k=m}^{2 m-1}\right) \int_{-1}^{1}\left|\Delta_{i}^{k}(u h) f(x)\right| d u \cdot\left|\frac{h}{\varepsilon}\right|^{2 m-1-k} \\
& \times \max _{|v| \leqslant 2 m-1}\left(\bar{\eta}+\delta_{k}^{2 m-1} \eta\right)\left(\frac{\widetilde{r}(x)+v h e^{i}}{\varepsilon}\right) \\
= & C \sum_{j=0}^{2} S_{j}(x, h) .
\end{aligned}
$$

При $x \notin G S_{0}(x, h)=0$; при $x \in G$

$$
\int_{0}^{\varepsilon}\left|S_{0}(x, h)\right|^{q} h^{-s q} \frac{d h}{h} \leqslant C_{1}|f(x)|^{q} \varepsilon^{-s q} \chi_{U_{2 m \varepsilon}(\partial G)}(x) \leqslant C_{2} r(x)^{-s q}|f(x)|^{q} \chi_{U_{2 m \varepsilon}(\partial G)}(x) .
$$

Оценим $S_{1}(x, h)$. Пусть $\bar{\eta}\left(\widetilde{r}\left(x+v h e^{i}\right) / \varepsilon\right) \neq 0$. Тогда

$$
\frac{\varepsilon}{3}<\widetilde{r}\left(x+v h e^{i}\right)<\frac{2 \varepsilon}{3}, \quad \frac{\varepsilon}{6}<r\left(x+v h e^{i}\right)<\frac{2 \varepsilon}{3},
$$


так что при $|v| h<\varepsilon / 18,|w| h<\varepsilon / 18$ в силу неравенства треугольника имеем

$$
\frac{1}{9} \varepsilon<r(x)<\frac{13}{18} \varepsilon, \quad \frac{1}{18} \varepsilon<r\left(x+w h e^{i}\right)<\frac{7}{9} \varepsilon .
$$

Отсюда при $(2 m-1) h<\varepsilon / 18$ получаем

$$
\begin{aligned}
S_{1}(x, h) & \leqslant C_{1} \int_{|v|<2 m-1} r\left(x+v h e^{i}\right)^{-s}\left|f\left(x+v h e^{i}\right)\right| d v \varepsilon^{s}\left(\frac{h}{\varepsilon}\right)^{m} \chi_{U_{2 m \varepsilon}(\partial G)}(x) \\
& \leqslant C_{2} M_{j}\left(r^{-s} f\right)(x) \varepsilon^{s-m} h^{m} \chi_{U_{2 m \varepsilon}(\partial G)}(x) .
\end{aligned}
$$

Тогда при $18(2 m-1) \theta=1,(2 m-1) h<\varepsilon$

$$
\int_{0}^{\theta \varepsilon} S_{1}(x, h)^{q} h^{-s q} \frac{d h}{h} \leqslant C_{3}\left(M_{j}\left(r^{-s} f\right)(x)\right)^{q} \chi_{U_{2 m \varepsilon}(\partial G)}(x) .
$$

Из очевидной оценки

$$
S_{2}(x, h) \leqslant C_{4} \sum_{k=m}^{2 m-1} \int_{-1}^{1}\left|\Delta_{i}^{k}(u h) f(x)\right| d u \chi_{U_{2 m \varepsilon}(\partial G)}(x)
$$

следует, что

$$
\int_{0}^{\varepsilon /(2 m-1)} S_{2}(x, h)^{q} h^{-s q} \frac{d h}{h} \leqslant C_{5} \sum_{k=m}^{2 m-1} \int_{0}^{\varepsilon /(2 m-1)}\left(\widetilde{\Delta}_{i}^{k}(h) f(x)\right)^{q} h^{-s q} \frac{d h}{h} \chi_{U_{2 m \varepsilon}(\partial G)}(x) .
$$

Пусть теперь $\theta \varepsilon<h<h_{0}$. Имеем

$$
\begin{aligned}
& \widetilde{\Delta}_{i}^{2 m-1}(h) f_{(\varepsilon)}(x) \leqslant C_{1} \sum_{k=0}^{2 m-1} \int_{-1}^{1}\left|f\left(k+k u h e^{i}\right)\right| \eta\left(\frac{\widetilde{r}(x)+k u h e^{i}}{\varepsilon}\right) d u \\
& \leqslant C_{2}\left(|f(x)| \eta\left(\frac{\widetilde{r}(x)}{\varepsilon}\right)+\frac{1}{h} \int_{|u|<(2 m-1) h}\left|f\left(x+u e^{i}\right)\right| \eta\left(\frac{\widetilde{r}(x)+u e^{i}}{\varepsilon}\right) d u\right) .
\end{aligned}
$$

Отсюда

$$
\int_{\theta \varepsilon}^{h_{0}}\left(\widetilde{\Delta}_{i}^{2 m-1}(h) f_{(\varepsilon)}(x)\right)^{q} h^{-s q} \frac{d h}{h} \leqslant C_{3}\left(M_{j}\left(r^{-s} f\right)(x)\right)^{q} \chi_{U_{\varepsilon}(\partial G)}(x) .
$$

Из полученных оценок и ограниченности в $L_{p}$ максимального оператора по $x_{j}$ следует (6), что завершает доказательство леммы.

ЗАмечаниЕ 1. Доказательства лемм 1,2 проводятся в два шага. На первом из них устанавливается ограниченность оператора $\mathscr{E}_{0}$ из $\stackrel{*}{B}_{p q}^{s}(G)$ в $B_{p q}^{s}\left(\mathbb{R}^{n}\right)$ и из $\stackrel{*}{F_{p q}^{s}}(G)$ в $F_{p q}^{s}\left(\mathbb{R}^{n}\right)$. На втором же решаются задачи спектрального синтеза в этих пространствах. Для решения задач второго шага можно было бы воспользоваться результатами Ю.В. Нетрусова [10] (их доказательства приведены в [11]).

Введем следующую характеристику границы $\partial G$ области $G$ :

$$
\mu(h, t)=t^{-n} \sup _{a \in \partial G} \operatorname{mes}\left(U_{h}(\partial G) \cap B_{t}(a)\right), \quad h, t>0 .
$$

Отметим, что $\mu(h, t)$ обладает свойствами удвоения: при некотором $C>0$ при всех $h \in\left(0, h_{0}\right], t \in(0, T]$

$$
\mu(2 h, t) \leqslant C \mu(h, t), \quad \mu(h, 2 t) \leqslant C \mu(h, t) .
$$

Легко видеть также, что

$$
\bar{\mu}(h, t):=t^{-n} \sup _{a \in \mathbb{R}^{n}} \operatorname{mes}\left(U_{h}(\partial G) \cap B_{t}(a)\right) \leqslant C \mu(h, t) .
$$


ТЕОремА 1. Пусть область $G \subset \mathbb{R}^{n}$ обладает свойством гибкого конуса, $s>0$, $1 \leqslant p<\infty, 1 \leqslant q<\infty$,

$$
M(G, p, s)=\int_{0}^{1} u^{-s} \sup _{0<t \leqslant T} \mu(u t, t)^{1 / p} \frac{d u}{u}<\infty \quad \text { либо } \quad s<\frac{\sigma_{0}}{p},
$$

где $\sigma_{0}$ то же, что и в лемме 4. Тогда операторы

$$
\mathscr{E}_{0}: B_{p q}^{s}(G) \rightarrow \stackrel{\circ}{B}_{p q}^{s}\left(\mathbb{R}^{n}, G\right), \quad \mathscr{E}_{0}: B_{p \infty}^{s}(G) \rightarrow B_{p \infty}^{s}\left(\mathbb{R}^{n}\right)
$$

ограничены.

ДокАЗАТЕЛЬСТво. По лемме 4 первое из условий (7) выполнено при выполнении второго. В силу леммы 1 достаточно показать, что $B_{p q}^{s}(G)=\stackrel{*}{B} p_{p q}(G)$, т.е. установить оценку

$$
\left(\int_{0}^{h_{0}}\left\|f \mid L_{p}\left(G \cap U_{h}(\partial G)\right)\right\|^{q} h^{-s q} \frac{d h}{h}\right)^{1 / q} \leqslant C\left\|f \mid B_{p q}^{s}(G)\right\| .
$$

Воспользуемся интегральньц представлением функции через разности $[6$, гл. 7 , формула (75)]:

$$
\begin{aligned}
f(x)=f_{T}(x)+ & \int_{0}^{T} \sum_{i=1}^{n} \int_{\mathbb{R}^{n}} \int_{-\infty}^{\infty} t^{-2-n} \Phi_{i}\left(\frac{y}{t}, \frac{\rho(t, x)}{t}\right) \zeta_{i}\left(\frac{u}{t}-\frac{\rho_{i}(t, x)}{2 t}, \frac{1}{2} \rho_{i}^{\prime}(t, x)\right) \\
& \times \Delta_{i}^{m}(\gamma u, G) f\left(x+y+u e^{i}\right) d u d y d t .
\end{aligned}
$$

Здесь

$$
\begin{gathered}
f_{T}(x)=T^{-n} \int \Phi_{0}\left(\frac{y}{T}, \frac{\rho(T, x)}{T}\right) f(x+y) d y, \\
\Phi_{i} \in C\left(\mathbb{R}^{n} \times \mathbb{R}^{n}\right), \quad \Phi_{i}(\cdot, z) \in C_{0}^{\infty}\left(\mathbb{R}^{n}\right) \quad \text { при } z \in Q_{0}, \quad i=0,1, \ldots, n, \\
\int \Phi_{0}(y, z) d y=1 \quad \text { при } z \in Q_{0}, \\
\zeta_{i} \in C\left(\mathbb{R}^{1} \times \mathbb{R}^{1}\right), \quad \zeta_{i}(\cdot, v) \in C_{0}^{\infty}\left(\mathbb{R}^{1}\right) \quad \text { при }|v| \leqslant 1 .
\end{gathered}
$$

При этом supp $\Phi_{0}(\cdot, z) \subset\{x:|x-z|<\delta\}$,

$$
\begin{gathered}
\operatorname{supp} \Phi_{i}(\cdot, z) \subset\left\{x:\left|x-z+\frac{1}{2} z_{i} e^{i}\right|<\delta\right\} \quad i=1, \ldots, n, \\
\operatorname{supp} \zeta_{i}(\cdot, v) \subset\{u:|u|<\delta\},
\end{gathered}
$$

где $\delta>0, \gamma>0$ столь малы, что представление (10) в точке $x \in G$ построено по сужению $f$ на гибкий конус $x+\bigcup_{0<t \leqslant T}\left(\rho(t, x)+t \delta_{0} Q_{0}\right) \subset G$.

Оценим $|f(x)|$ при $x \in E_{h}:=G \cap U_{h}(\partial G)$ с помощью представления (10):

$$
\begin{aligned}
|f(x)| \leqslant & T^{-n} \int \Psi\left(\frac{y}{T}\right)|f(x-y)| d y \\
& +\left(\int_{0}^{h}+\int_{h}^{T}\right) \int t^{-n+s} \Psi\left(\frac{y}{t}\right) t^{-s} \sum_{i=1}^{n} \widetilde{\Delta}_{i}(t, G) f(x-y) d y \frac{d t}{t} \\
= & \left(\Psi_{T} *|f|\right)(x)+\left(\int_{0}^{h}+\int_{h}^{T}\right) t^{s}\left(\Psi_{t} * g(\cdot, t)\right)(x) \frac{d t}{t}
\end{aligned}
$$


где $\Psi \in C_{0}\left(\mathbb{R}^{n}\right), \Psi \geqslant 0, g(x, t)=t^{-s} \sum_{i=1}^{n} \widetilde{\Delta}_{i}(t, G) f(x)$, функцию $f$ считаем продолженной нулем на $\mathbb{R}^{n} \backslash G$.

Пусть $\Psi(x)=0$ при $|x|>r$. С помощью неравенства Гёльдера имеем

$$
\begin{aligned}
\left|\left(\Psi_{t} * f\right)(x)\right| & \leqslant\left(\int \chi\left(\frac{x-y}{r t}\right)|f(y)|^{p} d y\right)^{1 / p}\left\|\Psi_{t} \mid L_{p^{\prime}}\right\| \\
& \leqslant C t^{-n / p}\left(\int \chi\left(\frac{x-y}{r t}\right)|f(y)|^{p} d y\right)^{1 / p}
\end{aligned}
$$

Из (11) с помощью (12) и неравенства Минковского получаем

$$
\begin{aligned}
\left\|f \mid L_{p}\left(E_{h}\right)\right\| \leqslant & C\left(\iint_{E_{h}} \chi\left(\frac{x-y}{r T}\right)|f(y)|^{p} d x d y\right)^{1 / p}+2^{m} \int_{0}^{h} t^{s}\|\Psi\|_{1}\|g(\cdot, t)\|_{p} \frac{d t}{t} \\
& +C \int_{h}^{T} t^{s-n / p}\left(\iint \chi\left(\frac{x-y}{r t}\right) g^{p}(y, t) d x d y\right)^{1 / p} \frac{d t}{t} \\
\leqslant & C_{1} \mu(h, T)^{1 / p}\|f\|_{p}+C_{1} \int_{0}^{h} t^{s}\|g(\cdot, t)\|_{p} \frac{d t}{t} \\
& +C_{1} \int_{h}^{T} t^{s} \mu(h, t)^{1 / p}\|g(\cdot, t)\|_{p} \frac{d t}{t}
\end{aligned}
$$

\section{Отсюда}

$$
\begin{aligned}
& \left(\int_{0}^{h_{0}}\left\|f \mid L_{p}\left(E_{h}\right)\right\|^{q} h^{-s q} \frac{d h}{h}\right)^{1 / q} \\
& \leqslant C\left(\int_{0}^{h_{0}} \mu(h, T)^{q / p} h^{-s q} \frac{d h}{h}\right)^{1 / q}\|f\|_{p}+C\left(\int_{0}^{h_{0}}\left(\int_{0}^{h} t^{s}\|g(\cdot, t)\|_{p} \frac{d t}{t}\right)^{q} h^{-s q} \frac{d h}{h}\right)^{1 / q} \\
& \quad+C\left(\int_{0}^{h_{0}}\left(\int_{h}^{T} t^{s} \mu(h, t)^{1 / p}\|g(\cdot, t)\|_{p} \frac{d t}{t}\right)^{q} h^{-s q} \frac{d h}{h}\right)^{1 / q} \\
& =C\left(L_{0}(f)+L_{1}(f)+L_{2}(f)\right)
\end{aligned}
$$

В силу монотонности $\mu(h, T)$ и неравенства Иенсена

$$
\begin{aligned}
L_{0}(f) & \leqslant\left(\sum_{k \leqslant \log _{2} h_{0}} \mu\left(2^{k}, T\right)^{q / p} \int_{2^{k}}^{2^{k+1}} h^{-s q} \frac{d h}{h}\right)^{1 / q}\|f\|_{p} \\
& \leqslant \sum_{k \leqslant \log _{2} h_{0}} \mu\left(2^{k}, T\right) 2^{-s k}\|f\|_{p} \leqslant C_{1} M(G, p, s)\|f\|_{p} .
\end{aligned}
$$

В силу неравенства Харди

$$
L_{1}(f) \leqslant C_{2}\left(\int_{0}^{h_{0}}\|g(\cdot, t)\|_{p}^{q} \frac{d t}{t}\right)^{1 / q}
$$


В $L_{2}(f)$ заменим $t$ на $h u$ и воспользуемся неравенством Минковского для интегралов:

$$
\begin{aligned}
L_{2}(f) & \leqslant \int_{1}^{\infty} u^{s}\left(\int_{0}^{T / u} \mu(h, h u)^{q / p}\|g(\cdot, h u)\|_{p}^{q} \frac{d h}{h}\right)^{1 / q} \frac{d u}{u} \\
& =\int_{1}^{\infty} u^{s}\left(\int_{0}^{T} \mu\left(\frac{t}{u}, t\right)^{q / p}\|g(\cdot, t)\|_{p}^{q} \frac{d t}{t}\right)^{1 / q} \frac{d u}{u} \\
& \leqslant \int_{0}^{1} v^{-s} \sup _{0 \leqslant t \leqslant T} \mu(v t, t)^{1 / p} \frac{d v}{v}\left(\int_{0}^{T}\|g(\cdot, t)\|_{p}^{q} \frac{d t}{t}\right)^{1 / q} \\
& =M(G, p, s)\left(\int_{0}^{T}\|g(\cdot, t)\|_{p}^{q} \frac{d t}{t}\right)^{1 / q} .
\end{aligned}
$$

Полученные оценки $L_{k}(f), k=0,1,2$, в силу (13) приводят к (9), а значит, к утверждению теоремы при $q<\infty$. Доказательство ограниченности второго оператора в $(8)$ аналогично.

Для доказательства аналога теоремы 1 для $F_{p q}^{s}$-пространств нам понадобятся некоторые леммы.

Лемма 3 (В.С. Рычков). Пусть $E \subset \mathbb{R}^{n}, \operatorname{mes} E=0, r(x)=\operatorname{dist}(x, E), 0<\sigma<n$, $0<T \leqslant \infty$,

$$
\sup _{\substack{x \in E \\ 0<R<T}}\left(R^{\sigma-n} \int_{|y-x|<R} \frac{d y}{r(y)^{\sigma}}\right)<\infty .
$$

Тогда при $1<p<\infty, 0 \leqslant s<\sigma / p$ оператор

$$
K f(x)=r(x)^{-s} \int_{r(x)<|y|<T} f(x+y) \frac{d y}{|y|^{n-s}}
$$

ограничен в $L_{p}\left(\mathbb{R}^{n}\right)$.

ДокАЗАТЕЛЬСтво. Оператор $K$ является аналогом одномерного оператора Харди

$$
\varphi \mapsto t^{-s} \int_{t}^{\infty} \varphi(u) \frac{d u}{u^{1-s}}
$$

и предлагаемое доказательство проводится по плану известного доказательства ограниченности последнего в $L_{p}((0, \infty))$. Ради простоты записи будем считать $T=\infty$.

С помощью неравенства Гёльдера имеем

$$
\begin{aligned}
|K f(x)|^{p} \leqslant & r(x)^{-s p} \int_{|y|>r(x)}|f(x+y)|^{p} r(x+y)^{\sigma p / p^{\prime}} \frac{d y}{|y|^{n-s-\gamma p}} \\
& \times\left(\int_{|y|>r(x)} \frac{d y}{r(x+y)^{\sigma}|y|^{n-s+\gamma p^{\prime}}}\right)^{p / p^{\prime}}
\end{aligned}
$$

где $1 / p+1 / p^{\prime}=1, \gamma=\sigma\left(1 / p-1 / p^{\prime}\right)-s / p$, так что $-s+\gamma p^{\prime}=-\sigma+\varepsilon$, где $\varepsilon=\sigma-s+\gamma p^{\prime}=$ $p^{\prime}(\sigma / p-s)>0$. Второй интеграл в (16) оценим с помощью справедливого при любом $\varepsilon>0$ неравенства

$$
I_{\varepsilon}(x)=\int_{|y|>r(x) / 2} \frac{d y}{r(x+y)^{\sigma}|y|^{n-\sigma+\varepsilon}} \leqslant C_{\varepsilon} r(x)^{-\varepsilon},
$$


где $C_{\varepsilon}$ не зависит от $x \in \mathbb{R}^{n}$. Оно устанавливается следующим образом:

$$
I_{\varepsilon}(x) \leqslant \sum_{j=-1}^{\infty} \int_{2^{j} r(x)<|y|<2^{j+1} r(x)} \frac{d y}{r(x+y)^{\sigma}}\left(2^{j} r(x)\right)^{\sigma-n-\varepsilon} .
$$

Пусть для данного $x \in \mathbb{R}^{n} \backslash E$ точка $x^{0} \in \bar{E}$ такова, что $\left|x-x^{0}\right| \leqslant r(x)$. Тогда с помощью (14) имеем

$$
\begin{aligned}
\int_{|y|<2^{j+1} r(x)} \frac{d y}{r(x+y)^{\sigma}} & =\int_{|y-x|<2^{j+1} r(x)} \frac{d y}{r(y)^{\sigma}} \leqslant \int_{\left|y-x^{0}\right|<2^{j+2} r(x)} \frac{d y}{r(y)^{\sigma}} \\
& \leqslant C\left(2^{j+2} r(x)\right)^{n-\sigma} .
\end{aligned}
$$

Применяя последнюю оценку к интегралам в (18), приходим к (17). Из (16) и (17) с $\varepsilon=p^{\prime}(\sigma / p-s)>0$ следует, что

$$
\begin{aligned}
|K f(x)|^{p} & \leqslant C_{\varepsilon} r(x)^{-\sigma} \int_{|y|>r(x)}|f(x+y)|^{p} r(x+y)^{\sigma p / p^{\prime}} \frac{d y}{|y|^{n-s-\gamma p}} \\
& =C_{\varepsilon} r(x)^{-\sigma} \int_{|z-x|>r(x)}|f(z)|^{p} r(z)^{\sigma p / p^{\prime}} \frac{d z}{|z-x|^{n-s-\gamma p}} .
\end{aligned}
$$

Отсюда

$$
\|K f\|_{p}^{p} \leqslant C_{\varepsilon} \int|f(z)|^{p} r(z)^{\sigma p / p^{\prime}} J(z) d z
$$

где

$$
J(z)=\int_{|x-z|>r(x)} \frac{d x}{r(x)^{\sigma}|x-z|^{n-\sigma+\sigma p / p^{\prime}}} .
$$

Так как $|x-z|>r(x)$ влечет $r(z) \leqslant r(x)+|x-z|<2|x-z|$, то, используя (17), при $\lambda=\sigma p / p^{\prime}$ имеем $J(z) \leqslant I_{\lambda}(z) \leqslant C_{\lambda} r(z)^{-\lambda}$. Отсюда и из (19) следует утверждение леммы.

Далее снова считаем $r(x)=\operatorname{dist}(x, \partial G)$.

Следуя рассуждениям Троценко [12], К. О. Бесов показал, что справедлива следующая

ЛЕмма 4. Пусть область $G \subset \mathbb{R}^{n}$ обладает свойством гибкого конуса с параметрами $\delta_{0}, T>0$ (см. определение 2$), \sigma_{0}=-\log _{2}\left(1-\left(\delta_{0} / 10\right)^{n}\right)$. Тогда

$$
\sup _{0<t \leqslant T} \mu(u t, t) \leqslant C\left(\delta_{0}, T\right) u^{\sigma_{0}}, \quad \sup _{\substack{x \in \mathbb{R}^{n} \\ 0<R<R_{0}}} R^{\sigma-n} \int_{|y-x|<R} \frac{d y}{r(y)^{\sigma}} \leqslant C\left(\delta_{0}, T, R_{0}, \sigma\right)
$$

при всех $\sigma \in\left(0, \sigma_{0}\right)$.

Отметим, что лемма 4 близка по форме лемме 6 из [13], базирующейся на результате из [12].

СлЕдСтВИЕ. Пусть область $G \subset \mathbb{R}^{n}$ обладает свойством гибкого конуса. Тогда при $1<p<\infty$ onepamop

$$
K_{0} f(x)=\int_{r(x)<|y|<T} f(x+y) \frac{d y}{|y|^{n}}
$$

ограничен в $L_{p}\left(\mathbb{R}^{n}\right)$. 
ТЕОрема 2. Пусть область $G \subset \mathbb{R}^{n}$ обладает свойством гибкого конуса, $p, q \in$ $(1, \infty), s>0,0<T<\infty$. Для ограниченности оператора

$$
\mathscr{E}_{0}: F_{p q}^{s}(G) \rightarrow \stackrel{\circ}{F}_{p q}^{s}\left(\mathbb{R}^{n}, G\right)
$$

достаточно выполнения какого-либо из следующих трех условий:

1) onepamop

$$
K_{s} f(x)=r(x)^{-s} \int_{r(x)<|y|<T} f(x+y) \frac{d y}{|y|^{n-s}}
$$

ограничен в $L_{p}\left(\mathbb{R}^{n}\right)$;

2) при некотором $\sigma \in(s p, n)$

$$
\sup _{\substack{x \in \partial G \\ 0<R<T}}\left(R^{\sigma-n} \int_{|y-x|<R} \frac{d y}{r(y)^{\sigma}}\right)<\infty
$$

3) $0<s<\sigma_{0} / p$, где $\sigma_{0}$ то же, что и в лемме 4 .

ДокАЗАтЕльство. Условие 2) влечет условие 1) в силу леммы 3 ; условие 3 ) влечет условие 2) в силу леммы 4. Поэтому для доказательства теоремы достаточно установить ограниченность оператора (20) при условии (21).

В силу леммы 2 достаточно установить, что $F_{p q}^{s}(G)=\stackrel{*}{F}_{p q}^{s}(G)$, т.е. получить оценку

$$
\left\|r^{-s} f\left|L_{p}\left(G \cap U_{h_{0}}(\partial G)\right)\|\leqslant C\| f\right| F_{p q}^{s}(G)\right\|
$$

Доопределим функцию $f$ нулем на $G$, и пусть $h_{0} \leqslant T$. В силу (11)

$$
r(x)^{-s}|f(x)| \leqslant C\left(N_{0} f(x)+N_{1} g(x)+N_{2} g(x)\right),
$$

где $N_{0} f(x)=r(x)^{-s}\left(\chi_{R T} *|f|\right)(x)$,

$$
\begin{gathered}
N_{1} g(x)^{q}=\int_{0}^{h_{0}}\left(\int_{0}^{h} t^{s}\left(\Psi_{t} * g(\cdot, t)\right)(x) \frac{d t}{t}\right)^{q} h^{-s q} \frac{d h}{h}, \\
N_{2} g(x)^{q}=\int_{r(x) /(m+1)}^{h_{0}}\left(\int_{h}^{T} t^{s}\left(\Psi_{t} * g(\cdot, t)\right)(x) \frac{d t}{t}\right)^{q} h^{-s q} \frac{d h}{h} .
\end{gathered}
$$

Здесь $g(x, t)$ то же, что в $(11), \chi_{t}(x)=t^{-n} \chi(x / t)$, а число $R \geqslant 1$ взято столь большим, что $\operatorname{supp} \Psi \subset B_{R}(0)$. Оценка

$$
\left\|N_{0} a\right\|_{p}^{p} \leqslant C_{1} \sup _{z} \int_{|x-z|<R} r(x)^{-s p} d x\|f\|_{p}^{p} \leqslant C_{2}\|f\|_{p}^{p}
$$

вытекает из (15), если взять там $\chi((x-z) /(R+T))$ в качестве $f(x)$.

Ограниченность оператора

$$
N_{1}: L_{p}\left(\mathbb{R}^{n}, L_{q}^{*}\left(0, h_{0}\right)\right) \rightarrow L_{p}\left(\mathbb{R}^{n}\right)
$$

установлена в [6, лемма 29.5]. 
Остается доказать ограниченность оператора

$$
N_{2}: L_{p}\left(\mathbb{R}^{n}, L_{q}^{*}\left(0, h_{0}\right)\right) \rightarrow L_{p}\left(\mathbb{R}^{n}\right),
$$

т.е. ограниченность оператора

$$
A: L_{p}\left(\mathbb{R}^{n}, L_{q}^{*}(0, T)\right) \rightarrow L_{p}\left(\mathbb{R}^{n}, L_{q}^{*}\left(0, h_{0}\right)\right),
$$

где

$$
A g(x, h)=\chi\left(\frac{r(x)}{h}\right) h^{-s} \int_{h}^{T} t^{s}\left(\Psi_{t} * g\right)(x, t) \frac{d t}{t} .
$$

Здесь свертка $\Psi_{t}$ с $g(x, t)$ взята лишш по $x$. Можно далее считать $h_{0}=T$.

Мы установим ограниченность при каждых $p, q \in(1, \infty)$ оператора

$$
A: L_{p}\left(G, L_{q}^{*}(0, T)\right) \rightarrow L_{p}\left(\mathbb{R}^{n}, M_{q}^{*}\left(0, h_{0}\right)\right)
$$

где $M_{q}^{*}\left(0, h_{0}\right)$ - пространство Марцинкевича на интервале $\left(0, h_{0}\right)$ по мере $d h / h$. Тогда ограниченность $A$ будет являться следствием интерполяционной теоремы Марцинкевича и интерполящионной теоремы $\left(L_{p}\left(B_{0}\right), L_{p}\left(B_{1}\right)\right)=L_{p}\left(B_{\theta}\right)$ (см., например, [14, п. 1.18.4]).

Зафиксируем $x \in \mathbb{R}^{n}$ и оценим квазинорму $A g(x, \cdot)$ в $M_{q}^{*}$, т.е.

$$
\begin{aligned}
\left\|A g(x, \cdot) \mid M_{q}^{*}\right\| & =\sup _{\lambda>0} \lambda \operatorname{mes}^{1 / q}\{h: A g(x, h)<\lambda\} \\
& =\sup _{0<\lambda<r} \lambda \operatorname{mes}^{1 / q}\left[r, h_{\lambda}\right]=\sup _{0<\lambda<r} \lambda \ln \frac{h_{\lambda}}{r},
\end{aligned}
$$

где $r=r(x), \mathrm{a} h_{\lambda} \in[r, T]$ взято из условия $A g(x, \lambda)=\lambda$. Очевидно,

$\left\|A g(x, \cdot) \mid M_{q}^{*}\right\|=\sup _{r \leqslant h \leqslant T} S(h), \quad$ где $S(h)=\ln ^{1 / q}\left(\frac{h}{r}\right) h^{-s} \int_{h}^{T} \chi\left(\frac{r}{h}\right) t^{s}\left(\Psi_{t} * g\right)(x, t) \frac{d t}{t}$.

Если $\varphi(h)=\ln ^{1 / q}(h / r) h^{-s}$, то

$$
\varphi^{\prime}(h)=\frac{1}{q} \ln ^{1 / q-1}\left(\frac{h}{r}\right) h^{-1-s}\left(\frac{1}{q}-s \ln \left(\frac{h}{r}\right)\right)
$$

и $S(h)$ при $h>r e^{1 /(q s)}$ убывает, как произведение двух убывающих множителей. Поэтому

$$
\sup _{r \leqslant h \leqslant T} S(h)=\sup _{r \leqslant h \leqslant e^{1 /(q s)} r} S(h) .
$$

Но $\ln (h / r) \leqslant C$ при $h \in\left[r, e^{1 /(q s)} r\right]$, так что

$$
\left\|A g(x, \cdot) \mid M_{q}^{*}\right\| \leqslant C A g(x, r(x)) .
$$

Положим

$$
g(x)=\left(\int_{0}^{T} g(x, t)^{q} \frac{d t}{t}\right)^{1 / q}
$$


и, применяя неравенство Гёльдера по $t$, получим $(r=r(x))$

$$
\begin{aligned}
A g(x, r) & \leqslant C r^{-s} \int \frac{g(x+y) d y}{(r+|y|)^{n-s}} \\
& \leqslant C_{1} r^{-n} \int_{|y|<r} g(x+y) d y+C_{1} r^{-s} \int_{r<|y|<T_{1}} \frac{g(x+y) d y}{|y|^{n-s}} \\
& \leqslant C_{1} M g(x)+C_{1} K_{s} g(x) .
\end{aligned}
$$

Здесь $M$ - максимальньй оператор Харди-Литтлвуда, ограниченный в $L_{p}\left(\mathbb{R}^{n}\right), K_{s}-$ оператор (21), ограниченньй в $L_{p}\left(\mathbb{R}^{n}\right)$ по условию теоремы.

Отсюда и из (28) вытекает (27), а следовательно, и (26). Оценки (24)-(26) в силу (23) влекут (22), чем и завершают доказательство теоремы 2.

ЗАмЕчаниЕ 2 . Оценки (9) и $(22)$ в случае $n=1, G=(0, \infty)$ известны (см. [15, п. 4.3.1]).

ПримеР 1. Пусть область $G \subset \mathbb{R}^{n}$ удовлетворяет условию конуса, т.е. существуют числа $T, \delta_{0}>0$ такие, что для любого $x \in G$ найдется единичный вектор $e=e(x)$ такой, что

$$
x+\bigcup_{0 \leqslant t \leqslant T}\left(t e(x)+t \delta_{0} Q_{0}\right) \subset G .
$$

За счет уменьшения $\delta_{0}$ функцию $x \mapsto e(x)$ можно считать конечнозначной: $e(x) \in\left\{e^{j}\right\}_{j=1}^{J}$, $\left|e^{j}\right|=1$.

Тогда область $G$ представима в виде

$$
G=\bigcup_{j=1}^{J} G^{(j)}=\bigcup_{j=1}^{J}\left(G^{(j)}+V_{j}\right)
$$

где $G^{(j)}$ - открытые множества, $V_{j}=\bigcup_{0<t \leqslant T}\left(t e^{j}+t \delta_{1} Q_{0}\right)$. Соответственно, граница $\partial G$ представляется в виде $\partial G=\bigcup_{0<t \leqslant T}^{J} S_{j}$, причем $S_{j}+V_{j} \subset G$.

Очевидно, что внутри каждого шара $B_{t}(a), 0<t<T, a \in \mathbb{R}^{n}$, произвольная прямая, параллельная $e^{j}$, пересекает $S_{j}$ не более чем в одной точке, а ее пересечение с $U_{h}\left(S_{j}\right) \cap B_{t}(a), 0<h \leqslant t$, содержится в интервале, длина которого не превосходит $C h$. Следовательно, $\mu(h, t) \leqslant C_{1} h / t$. При $0<s<1 / p$ вьполнено условие $(7)$ :

$$
M(G, p, s) \leqslant C \int_{0}^{1} u^{-s+1 / p} \frac{d u}{u}<\infty .
$$

В силу теоремы 1 оператор продолжения $\mathscr{E}_{0}$ из (8) ограничен при $0<s<1 / p$. Легко убедиться, что условие 2$)$ теоремы 2 вьполнено при любом $\sigma \in(0,1)$. В силу теоремы 2 оператор продолжения $\mathscr{E}_{0}$ из $(20)$ ограничен при $0<s<1 / p$.

ПримеР 2. Пусть $n=2,(r, \varphi)$ - полярные координаты,

$$
G=\left\{(r, \varphi): e^{-2 \varphi}<r<e^{-\varphi}, 0<\varphi<\infty\right\}, \quad 0<s<\frac{1}{p} .
$$

Легко убедиться, что $\mu(h, t) \leqslant C h / t$, условие $(7)$ вьполнено и в силу теоремы 1 оператор продолжения $\mathscr{E}_{0}$ из (8) ограничен. 
ПРИмеР 3. Пусть $G=\mathbb{R}^{n} \backslash \Gamma$,

$$
\Gamma=\left\{x: x=\left(x^{\prime}, x^{\prime \prime}\right), x^{\prime}=\left(x_{1}, \ldots, x_{k}\right) \in \mathbb{R}^{k}, x^{\prime \prime}=\varphi\left(x^{\prime}\right)\right\},
$$

отображение $\varphi$ удовлетворяет условию Липшица, $0<s<(n-k) / p$. Тогда $\mu(h, t) \leqslant$ $C(h / t)^{k}$, условие (7) вьполнено и оператор $\mathscr{E}_{0}$ из (8) ограничен. Условие 2) теоремы 2 выполнено при любом $\sigma \in(0, n-k)$, и оператор продолжения $\mathscr{E}_{0}$ из (20) ограничен. Ограниченность операторов $(8),(20)$ в этом примере вытекает также из [10].

\section{СПИСОК ЦИТИРОВАННОЙ ЛИТЕРАТУРЫ}

[1] Kuttner B. Some theorems on fractional derivatives // Proc. London Math. Soc. 1953. V. 3. P. 480-497.

[2] Никольский С. М. Об одном свойстве классов $H_{p}^{(r)} / /$ Ann. Univ. Sci. Budapest. Eötvös Sect. Math. 1960/61. V. 3-4. P. 205-216.

[3] Яковлев Г. Н. Граничные свойства функций класса $W_{p}^{(l)}$ на областях с угловыми точками // Докл. АН СССР. 1961. Т. 140. №1. С. 73-76.

[4] Солонников В.А. Об одном классе функциональных пространств и об априорных оценках для решений некоторых краевых задач математической физики. Автореферат дисс. ... к. ф.-м.н. Л.: ЛГУ, 1961.

[5] Калябин Г. А. Теоремы о продолжении, мультипликаторах и диффеоморфизмах для обобщенных классов Соболева-Лиувилля в областях с липшицевой границей // Тр. МИАН. 1985. T. 172 . C. $173-186$.

[6] Бесов О.В., Ильин В. П., Никольский С. М. Интегральные представления функций и теоремы вложения. М.: Наука, 1996.

[7] Стейн И. Сингулярные интегралы и дифференциальные свойства функций. М.: Мир, 1973.

[8] Бурёнков В.И. О регуляризованном расстоянии // Тр. МИРЭА. Матем. Вып. 67. М.: МИРЭА, 1973. С. 113-117.

[9] Бесов О.В.О пространствах Соболева-Лиувилля и Лизоркина-Трибеля на области // Тр. МИАН. 1990. Т. 192 . С. 20-34.

[10] Нетрусов Ю. В. Спектральный синтез в пространствах гладких функций // Докл. РАН. 1992. T. 325. № 5. C. 923-925.

[11] Adams D.R., Hedberg L. I. Function Spaces and Potential Theory. Berlin: Springer, 1996.

[12] Троценко Д. А. Свойства областей с негладкой границей // Сиб. матем. ж. 1981. Т. 22. № 4. C. 221-224.

[13] Hajłasz P., Koskela P. Isoperimetric inequalities and imbedding theorems in irregular domains // J. London Math. Soc. (to appear).

[14] Трибель X. Теория интерполяции, функциональные пространства, дифференциальные операторы. М.: Мир, 1980.

[15] Schmeisser H.-J., Triebel H. Topics in Fourier Analysis and Function Spaces.. Leipzig: Geest \& Portig, 1987. 